\title{
Microcrystals of an organic fluorescent dye grown in the presence of various PAMAM dendrimers: Control of the morphology and optical properties
}

\author{
Franck Bertorelle, Fatima Al-Ali, and Suzanne Fery-Forgues ${ }^{\dagger}$ \\ Laboratoire des Interactions Moléculaires Réactivité Chimique et Photochimique, UMR CNRS 5623, \\ Université Paul Sabatier, 118 route de Narbonne, 31062 Toulouse cedex, France
}

\begin{abstract}
Microcrystals of a fluorescent dye, 4- $n$-octylamino-7-nitrobenzoxadiazole, were prepared by the reprecipitation method. Poly(amidoamine)dendrimers bearing anionic, cationic or neutral terminating groups were used as additives in the recrystallization medium. They directed the formation of thin plates or spindlelike microcrystals. The UV/vis absorption and emission properties were investigated on the microcrystal suspensions.
\end{abstract}

\section{INTRODUCTION}

Microcrystals of organic molecules are considered as advanced materials with promising technological applications in the field of photonics, nonlinear optics and electronics [1]. These tiny particles display original solid-state properties, which markedly differ from those of bulk crystals. For instance, their characteristics in UV/visible absorption [2-17] and emission [15-21] are closely related to their size. This behaviour must be distinguished from the conventional quantum confinement effect, widely studied for inorganic semiconductors with a size below $10 \mathrm{~nm}$ [22], and which is expected to be weak for organic compounds. Actually, the finite size effect has been reported to take place in submicrometer organic crystals, and is uniquely encountered in this type of compounds. According to Nakanishi and coworkers, it could be explained by the large proportion of surface molecules that are present in microcrystals, compared to bulk crystals. The electric field effect of the medium could interfere through these surface molecules. Alternatively, the loose molecular packing could reduce the coulombic interaction energies between molecules [17, 19]. Moreover, in microcrystals, the surface displays irregular molecular arrangements, and different lattices may co-exist [21]. It has been proposed that one or two types of excitons (free and self-trapped) are formed in organic microcrystals $[9,15,18,21,23,24]$. The exciton dynamics seems to depend on the size of the microcrystals, the site that receives the excitation beam, and the existence and concentration of crystal defects. However, quite few organic microcrystals have been studied so far. This can be explained by the difficulties linked to their preparation. Owing to the thermal instability of organic compounds, only a few

\footnotetext{
†E-mail: sff@chimie.ups-tlse.fr
}

methods can be used, and obtaining homogeneous populations of microcrystals with well-defined size and morphology is still a real challenge. We show here that the shape and size of microcrystals prepared by the reprecipitation method can be nicely tuned using differently-terminated dendrimers as additives. A comparison of the optical properties of the microcrystals obtained was then undertaken.

\section{RESULTS AND DISCUSSION}

The reprecipitation method is a very simple and convenient way to produce organic microcrystals [25, 26]. It consists in mixing a concentrated solution of an organic compound with a second solvent, in which the compound is insoluble. As a result, the organic compound precipitates and microcrystals may form as stable suspension. We recently used this method to prepare microcrystals of 4- $n$-octylamino-7-nitrobenz2-oxa-1,3-diazole (1) [27]. This dye belongs to the well-known nitrobenzoxadiazole (NBD) series, the amino derivatives of which usually display excellent absorption and fluorescence properties [28, 29]. To obtain<smiles>CCCCCCNc1ccc([N+](=O)[O-])c2nonc12</smiles>

microcrystals, a concentrated solution of dye $\mathbf{1}$ in ethanol was prepared, and then microamounts of this solution were rapidly injected into water. The dye concentration in the recrystallization medium was 

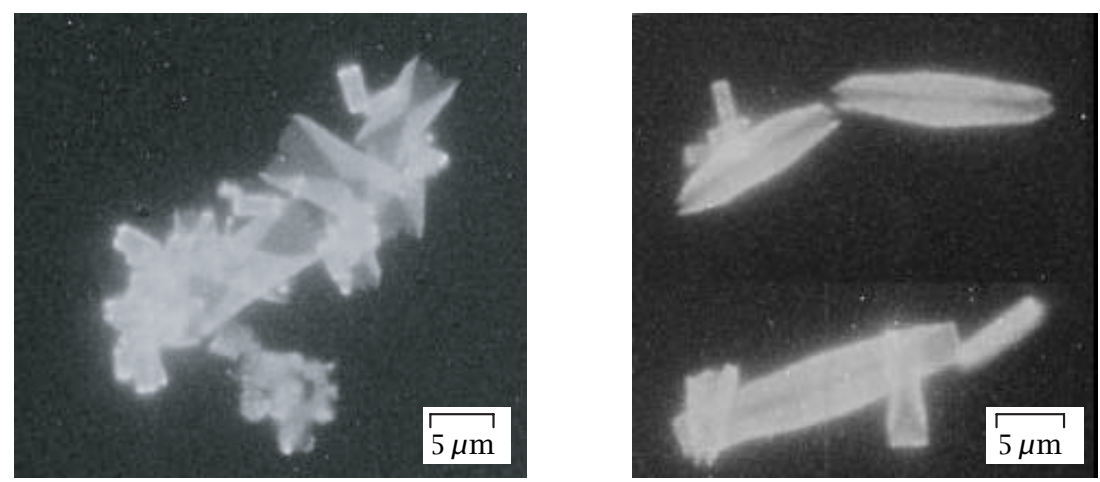

Figure 1. Fluorescence microscopy image of microcrystals of 1 grown in the presence of cationic G4 PAMAM dendrimer (left), and in the presence of neutral G4 glucose-persubstituted PAMAM dendrimer (right). Total dye concentration: $2 \times 10^{-5} \mathrm{M}$; Dendrimer concentration: $5 \times 10^{-5} \mathrm{M}$.

$2 \times 10^{-5} \mathrm{M}$, a value almost 40 times higher than the solubility threshold. The yellow solution discoloured, and the organic compound precipitated and crystallized as red agglomerated, irregular microcrystals. The whole process, monitored by UV/vis absorption spectroscopy, took about $1 \mathrm{~h}$. Subsequently, the possibility to control the size and shape of the particles was investigated. One strategy was to change the temperature of the crystallization medium, and the concentration of the injected solution [2-6, 14-18]. Another one was to introduce additives into the medium. These often are surfactants, which act as inhibitors, preventing one or many crystal faces from growing [2, 1113]. To our knowledge, macromolecules have not been used as additives in the reprecipitation method. However, in the field of inorganics, Naka and coworkers have recently shown that the crystallization mode of $\mathrm{CaCO}_{3}$ was modified upon introduction of an anionic dendrimer in the crystallization medium [30, 31]. This observation had never been extended to organic compounds. This prompted us to investigate the influence of a dendrimer upon the reprecipitation process of our dye. To do so, anionic carboxylate-terminated dendrimer of the PAMAM family, generation 3.5, was added to the aqueous crystallization medium, prior to injection of the dye solution. Unexpectedly, in the presence of $5 \times 10^{-5} \mathrm{M}$ dendrimer, the crystallization process was achieved within $10 \mathrm{~min}$. The suspension was pink, with a yellow glint, and the particles were invisible to the naked eye. The size and shape of the microcrystals were analysed by fluorescence microscopy, all the crystals emitting fluorescence above $580 \mathrm{~nm}$. They were rectangular, extremely thin, and displayed a characteristic Xshaped pattern at the centre. Three populations were observed, with sizes $40 \times 15,15 \times 8$, and $4 \times 2 \mu \mathrm{m}$ for the two largest sides. Contrary to what was observed in water, the crystals grown in the presence of dendrimer did not agglomerate [27].
The question that arises now is whether the dendrimer charge has an influence upon the microcrystallization process. So, the anionic dendrimer was replaced by a cationic PAMAM dendrimer, generation G4, bearing 64 primary amino groups at the periphery. In water, at neutral $\mathrm{pH}$, all the amino groups are protonated [32]. The recrystallization experiment was repeated with 1 in the presence of $5 \times 10^{-5}$ M G4 dendrimer. Results very similar to those observed with the anionic dendrimer were obtained, regarding the crystallization kinetics, and the morphology of the microcrystals (Figure 1, left). However, only two populations of microcrystals were detected, the largest measuring $12 \times 5 \mu \mathrm{m}$.

Since both anionic and cationic dendrimers have an influence upon the reprecipitation process, the influence of an equimolar mixture of these dendrimers (each at $2.5 \times 10^{-5} \mathrm{M}$ ) was also regarded. The kinetics was close to that obtained with only one dendrimer at $5 \times 10^{-5} \mathrm{M}$. However, it must be emphasised that only one population of small rectangular microcrystals was now observed, measuring about $5 \times 2 \mu \mathrm{m}$.

Finally, this work was extended to a neutral dendrimer, carrying out reprecipitation in the same conditions as above. A PAMAM dendrimer (G4) persubstituted by 64 glucose groups [33], was dissolved in water at a concentration of $5 \times 10^{-5} \mathrm{M}$. Again, the crystallization process was drastically accelerated compared with water, but it was slightly slower than with charged dendrimers, since it took about $15 \mathrm{~min}$ to reach completion. Most of the microcrystals exhibited a spindle-like shape, with a dark line in the middle (Figure 1, right). They measured about $15 \times 4 \mu \mathrm{m}$ for the two largest dimensions, and were very thin.

Blanks were performed using aqueous solutions of sodium acetate at a concentration of $3.2 \times 10^{-3} \mathrm{M}$, and 1,6-hexanediamine at $1.6 \times 10^{-3} \mathrm{M}$, which corresponds to the concentration of terminal groups in the 
dendrimer solutions. The crystallization rate was enhanced, but remained far below that obtained in the presence of charged dendrimers. In both cases, agglomerated microcrystals were produced. This indicates that the increase in ionic strength of the medium due to the presence of the terminal groups cannot account for the effect observed in the presence of charged dendrimers. Besides, the same experiment was performed in the presence of glucose $\left(3.2 \times 10^{-3} \mathrm{M}\right)$, and reprecipitation was found to be unchanged compared to water alone. Consequently, the fact that the functional groups are borne by a macromolecule seems to be a requirement for a strong effect to be observed upon reprecipitation.

For each experiment, the crystallization kinetics was monitored by UV/vis absorption spectroscopy. The kinetics profile gave evidence for a three-step mechanism, as previously established for $\mathbf{1}$ in water alone and in the presence of the G3.5 dendrimer [27]. These steps were attributed to the formation of small aggregates from the isolated NBD molecules, to their assembly in large aggregates, and to the formation of microcrystals. The same process seems to take place here. The presence of dendrimer accelerates each of these steps. It is interesting to note that each dendrimer investigated had an influence upon reprecipitation, whatever the nature of the peripheral groups. Since the NBD group displays sites of different polarities, interactions with oppositely charged dendrimers are possible. Actually, in our previous work, it was shown by UV/vis absorption and fluorescence spectroscopy that an interaction, probably of electrostatic nature, takes place between 1 and the carboxylic groups of the anionic dendrimer [27]. A similar effect was observed here with the amino groups, since the UV/vis absorption spectrum of 1 was strongly perturbed upon addition of 1,6-hexanediamine or cationic dendrimer to the solution. Moreover, it is known that amino-NBD dyes show particular affinity for hydroxyl groups [28], which suggests that a weak interaction can also take place with the glucose-terminated dendrimer. These interactions could lead to increased local concentration of NBD molecules near the dendrimer surface, directly influencing nucleation and crystal growth. Moreover, the dendrimers could act as stabilisers for the colloids of the NBD derivative, preventing them from coalescing.

This hypothesis is supported by the fact that a small amount of G3.5 dendrimer (17\% in weight) was detected by elemental analysis in dried microcrystals of $\mathbf{1}$, as mentioned in our previous work [27].

The optical properties of the different microcrystals in suspension in their native medium were investigated. The UV-visible absorption spectra were deconvoluted (Figure 2).

For the microcrystals obtained with the three pure dendrimers, the spectrum was composed of five bands. Bands $\mathrm{A}$ and $\mathrm{C}$ are quite close, respectively, to the

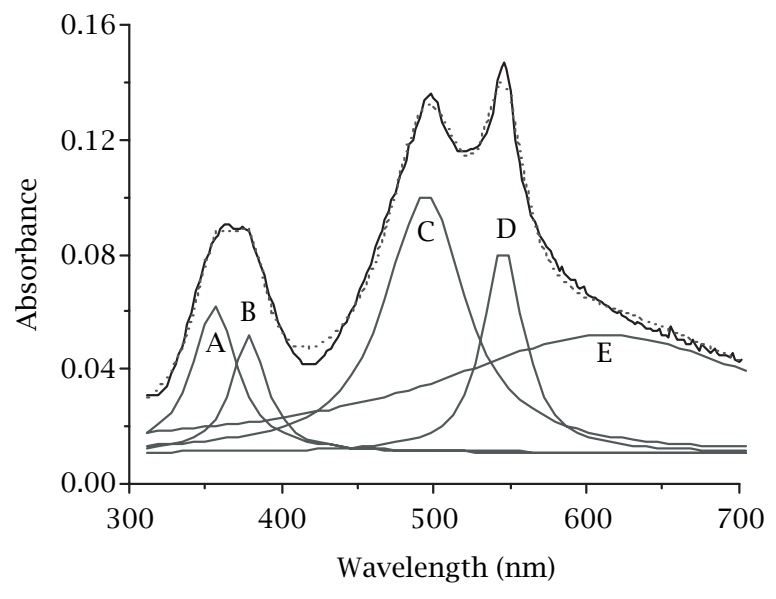

Figure 2. UV/vis absorption spectrum of microcrystals of $\mathbf{1}$ grown in the presence of G4 PAMAM dendrimer (top full line). Bands A to E were obtained by deconvolution, using 5 Lorentzian functions. The broken line is the sum of the calculated bands. Total dye concentration in the suspension: $2 \times 10^{-5} \mathrm{M}$; Dendrimer concentration: $5 \times 10^{-5} \mathrm{M}$.

$\pi-\pi^{*}$ and charge transfer bands of molecules 1 dissolved in water. They can be attributed to the contribution of individual molecules, i.e., Frenkle-like excitons. Bands B and D can be assigned to delocalized, chargetransfer (CT) excitons, which are considered to absorb at low energy. Band $\mathrm{E}$ was attributed to diffusion. From one set of microcrystals to another, bands A to E underwent only slight shifts in wavelength. Small differences were observed in their relative intensities. For the microcrystals obtained with the dendrimer mixture, a sixth band was found. It was centred at $466 \mathrm{~nm}$, and its intensity was not negligible.

The deconvolution of the emission spectra showed the existence of two bands, centred at around 575 and $630-665 \mathrm{~nm}$. By analogy with the fluorescence spectrum of the dissolved dye, the first band can be attributed to the free exciton, while the second band could arise from the CT exciton. The intensity ratio of the two bands was almost constant (around 80:20) for the microcrystals obtained in the presence of the pure dendrimers. In contrast, the first band was relatively less intense for the microcrystals grown in the dendrimer mixture. This result is quite surprising, since the intensity of the long-wavelength emission band is generally reported to increase when the microcrystal size increases [15, 18, 19].

The emission quantum yield was measured on the suspensions by exciting at $482 \mathrm{~nm}$. It was found to be around $3 \times 10^{-3}$ in every case. The decays were measured by collecting the emission at $576 \mathrm{~nm}$. They were monoexponential, and the lifetimes were found to be below $1 \mathrm{~ns}$. Therefore, it seems that the emission which arises from the free exciton is close to fluorescence. 
However, it must be noted that with the experimental set-up used, only a weak signal was recorded, and no satisfactory signals were obtained when gathering emissions at higher wavelengths. In these conditions, the possibility for a different lifetime to be associated to the second emission band cannot be ruled out.

\section{EXPERIMENTAL SECTION}

3.1. Materials. Compound $\mathbf{1}$ was synthesised as previously described [34]. Absolute ethanol was from Carlo Erba Reagenti. High-pressure demineralized water (resistivity $16 \mathrm{M} \Omega \mathrm{cm}$ ) was used. Anionic and cationic PAMAM starburst dendrimers were purchased from Aldrich and used without further purification. The glucose-persubstituted dendrimer was previously synthesised in the laboratory [33]. Sodium acetate was from Prolabo, 1,6-hexanediamine and glucose were from Acros.

3.2. Apparatus. UV/vis absorption spectra were recorded on a Hewlett-Packard 8452A diode array spectrophotometer. The measurements were conducted at $25^{\circ} \mathrm{C}$ in a thermostatted cell. Corrected steady-state fluorescence spectra were registered on a Photon Technology International (PTI) Quanta Master 1 spectrofluorometer. The fluorescence quantum yields $(\Phi)$ were determined using the classical formula: $\Phi_{\mathrm{X}}=\left(A_{\mathrm{S}} \times\right.$ $\left.F_{\mathrm{x}} \times n_{\mathrm{x}}{ }^{2} \times \Phi_{\mathrm{s}}\right) /\left(A_{\mathrm{x}} \times F_{\mathrm{S}} \times n_{\mathrm{s}}{ }^{2}\right)$ where $A$ is the absorbance at the excitation wavelength, $F$ the area under the fluorescence curve and $n$ the refraction index. Subscripts s and $x$ refer to the standard and to the sample of unknown quantum yield, respectively. Coumarin 6 in ethanol $(\Phi=0.78)$ was taken as the standard [35]. Fluorescence decay was measured with the stroboscopic technique utilising a Strobe Master fluorescence lifetime spectrometer from PTI. The excitation source $\left(\lambda_{\mathrm{ex}}=337 \mathrm{~nm}\right)$ was a flash lamp filled with a mixture of nitrogen and helium (30/70). Data were collected over 200 channels with a time-base of 0.1 ns per channel. Fluorescence decay was analysed using the monoexponential method software from PTI. The size and shape of the microcrystals were observed with a Zeiss MC80DX fluorescence microscope.

\section{CONCLUSION}

The use of various dendrimers as additives allowed us to obtain microcrystals of different size and shape. The optical properties of the microcrystals grown in the presence of pure dendrimers were quite similar, whether the crystals appear as platelets or as spindles. Only the microcrystals grown with the dendrimer mixture showed quite different characteristics. This can be related to the small size of the microcrystals, or to the presence of crystal imperfections. Another explanation could be the formation of a second type of molecular assembly, indistinguishable by fluorescence microscopy. It must be emphasised that all measurements were performed on microcrystal suspensions, so they give average values. Near field spectroscopy, which only considers one microcrystal, or a part of a microcrystal, could provide much more information [36]. Studies are underway in this direction, to get further insight into the microcrystals properties, and into the exact role played by the additive during the reprecipitation process.

\section{ACKNOWLEDGMENT}

Thanks are due to Dr I. Rico-Lattes for the gift of glucose-persubstituted dendrimer.

\section{REFERENCES}

[1] H. Oikawa, H. Kasai, and H. Nakanishi, in Anisotropic Organic Materials, ACS Symposium Ser. 798, (R. Glaser and P. Kasizynski, Eds.), American Chemical Society: Washington, DC, 2002, Chapter 12, pp. 169-178.

[2] H. Nakanishi and H. Katagi, Supramol. Sci. 5 (1998), 289.

[3] H. Kasai, H. Oikawa, S. Okada, and H. Nakanishi, Bull. Chem. Soc. Jpn. 71 (1998), 2597 and references cited.

[4] H. Katagi, H. Kasai, S. Okada, H. Oikawa, H. Matsuda, and H. Nakanishi, J. Macromol. Sci. Pure Appl. Chem. A 34 (1997), 2013.

[5] H. Katagi, H. Oikawa, S. Okada, H. Kasai, A. Watanabe, O. Ito, Y. Nozue, and H. Nakanishi, Mol. Cryst. Liq. Cryst. Sci. Tech., section A 314 (1998), 285.

[6] T. Onodera, H. Kasai, S. Okada, H. Oikawa, K. Mizuno, M. Fujitsuka, O. Ito, and H. Nakanishi, Opt. Mater. 21 (2002), 595.

[7] R. Iida, H. Kamatani, H. Kasai, S. Okada, H. Oikawa, H. Matsuda, A. Kakuta, and H. Nakanishi, Mol. Cryst. Liq. Cryst. 267 (1995), 95.

[8] H. Kasai, S. Okazaki, S. Okada, H. Oikawa, T. Adschiri, K. Arai, and H. Nakanishi, Mol. Cryst. Liq. Cryst. Sci. Tech., section B 24 (2000), 83.

[9] S. Mochizuki, M. Sasaki, and R. Ruppin, J. Phys.: Condens. Matter. 10 (1998), 2347.

[10] K. Baba, H. Kasai, S. Okada, H. Oikawa, and H. Nakanishi, Opt. Mater. 21 (2002), 591.

[11] T. Oshikiri, H. Kasai, H. Katagi, S. Okada, H. Oikawa, and H. Nakanishi, Mol. Cryst. Liq. Cryst. Sci. Tech., section A 337 (1999), 25.

[12] T. Onodera, T. Oshikiri, H. Katagi, H. Kasai, S. Okada, H. Oikawa, M. Terauchi, M. Tanaka, and H. Nakanishi, J. Crystal Growth 229 (2001), 586.

[13] X. Ji, Y. Ma, Y. Cao, X. Zhang, R. Xie, H. Fu, D. Xiao, and J. Yao, Dyes Pigm. 51 (2001), 87.

[14] H.-B. Fu, Y.-Q. Wang, and J.-N. Yao, Chem. Phys. Lett. 322 (2000), 327. 
[15] H.-B. Fu and J.-N. Yao, J. Am. Chem. Soc. 123 (2001), 1434.

[16] H. Kasai, Y. Yoshikawa, T. Seko, S. Okada, H. Oikawa, H. Mastuda, A. Watanabe, O. Ito, H. Toyotama, and H. Nakanishi, Mol. Cryst. Liq. Cryst. 294 (1997), 173 and references cited.

[17] H. Kasai, H. Kamatani, S. Okada, H. Oikawa, H. Matsuda, and H. Nakanishi, Jpn. J. Appl. Phys. 35 (1996), L221.

[18] H. Fu, D. Xiao, R. Xie, X. Ji, and J.-N. Yao, Can. J. Chem. 81 (2003), 7.

[19] H. Kasai, H. Kamatani, Y. Yoshikawa, S. Okada, H. Oikawa, A. Watanabe, O. Itoh, and H. Nakanishi, Chem. Lett. (1997), 1181.

[20] H. Oikawa, T. Mitsui, T. Onodera, H. Kasai, H. Nakanishi, and T. Sekiguchi, Jpn. J. Appl. Phys. 42 (2003), L111.

[21] A. H. Matsui, K. Mizuno, O. Nishi, Y. Matsushima, M. Shimizu, T. Goto, and M. Takeshima, Chem. Phys. 194 (1995), 167.

[22] P. Alivisatos, Science 271 (1996), 933.

[23] H. Nishimura, T. Yamaoka, K. Mizuno, M. Iemura, and A. Matsui, J. Phys. Soc. Jpn. 53 (1984), 3999.

[24] T. Fujino and T. Tahara, J. Phys. Chem. B 107 (2003), 5120.

[25] H. Oikawa, H. Kasai, and H. Nakanishi, in Anisotropic Organic Materials, ACS Symposium
Ser. 798, (R. Glaser and P. Kasizynski, Eds.), American Chemical Society: Washington, DC , 2002, Chapter 11, pp. 158-168.

[26] E. Van Keuren, E. Georgieva, and J. Adrian, Nanolett. 1 (2001), 141.

[27] F. Bertorelle, D. Lavabre, and S. Fery-Forgues, J. Am. Chem. Soc. 125 (2003), 6244.

[28] S. Fery-Forgues, J.-P. Fayet, and A. Lopez, J. Photochem. Photobiol. 70 (1993), 229.

[29] A. Chattopadhyay, Chem. Phys. Lipids 53 (1990), 1.

[30] K. Naka, Y. Tanaka, Y. Chujo, and Y. Ito, J. Chem. Soc. Chem. Commun. (1999), 1931.

[31] K. Naka, Y. Tanaka, and Y. Chujo, Langmuir 18 (2002), 3655.

[32] I. Lee, B. D. Athey, A. W. Wetzel, W. Meixner, and J. R. Baker, Macromol. 35 (2002), 4510.

[33] A. Schmitzer, E. Perez, I. Rico-Lattes, A. Lattes, and S. Rosca, Langmuir 15 (1999), 4397.

[34] F. Galinier, F. Bertorelle, and S. Fery-Forgues, C. R. Acad. Sci., Paris 4 (2001), 941.

[35] G. A. Reynolds and K. H. Drexhage, Opt. Commun. 13 (1975), 222.

[36] H. Yoshikawa and H. Masuhara, J. Photochem. Photobiol. C 1 (2000), 57. 


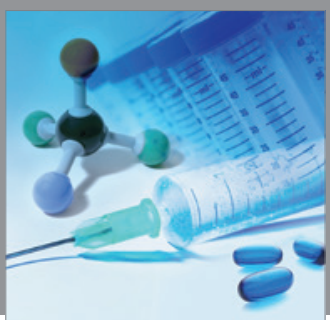

International Journal of

Medicinal Chemistry

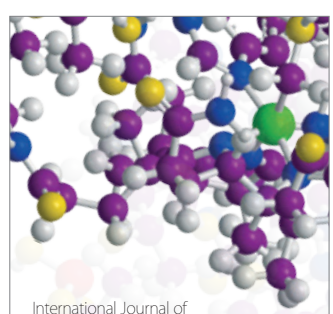

Carbohydrate Chemistry

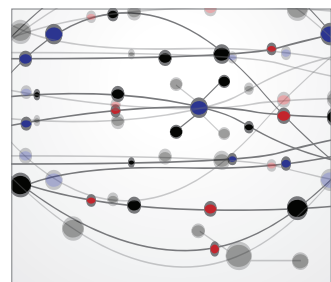

The Scientific World Journal
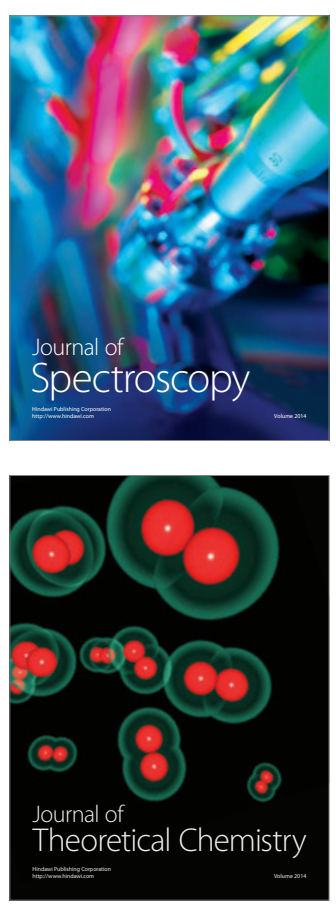
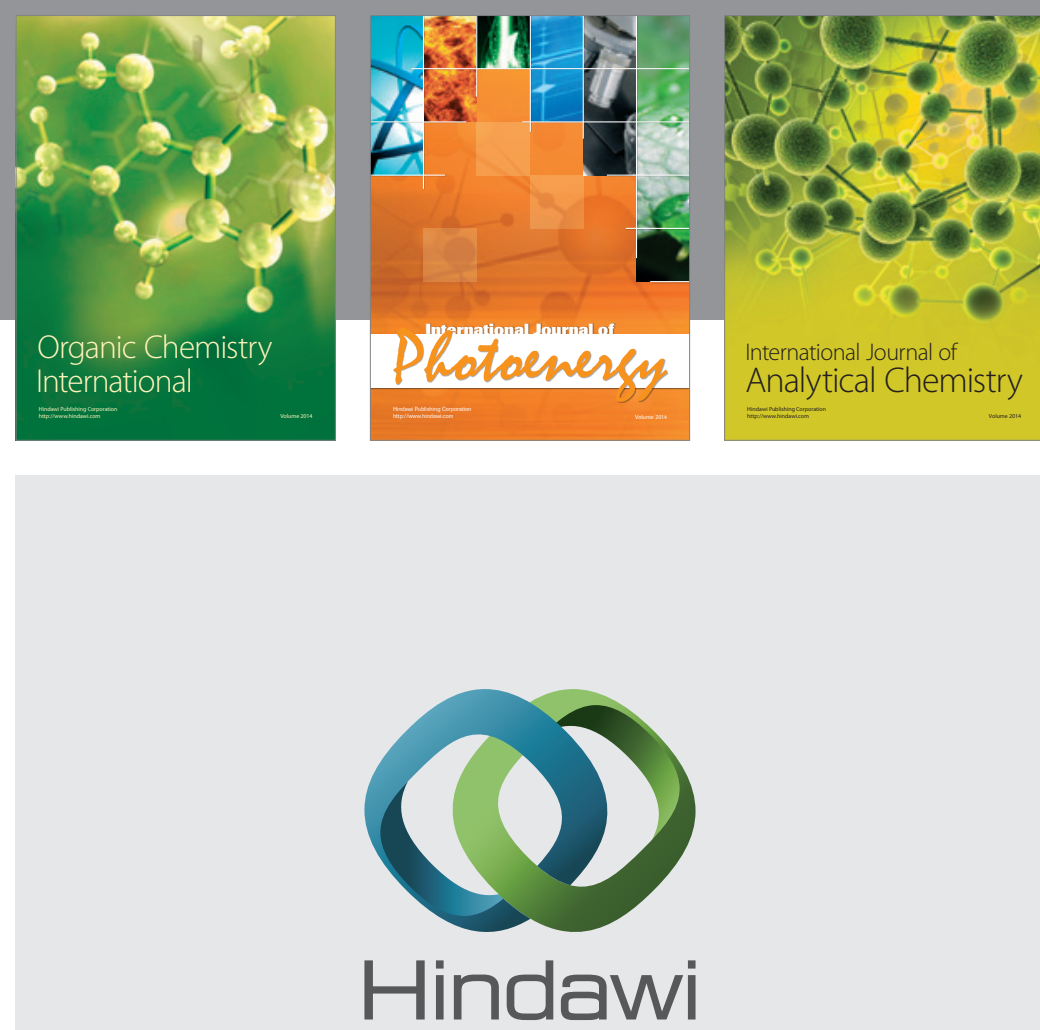

Submit your manuscripts at

http://www.hindawi.com
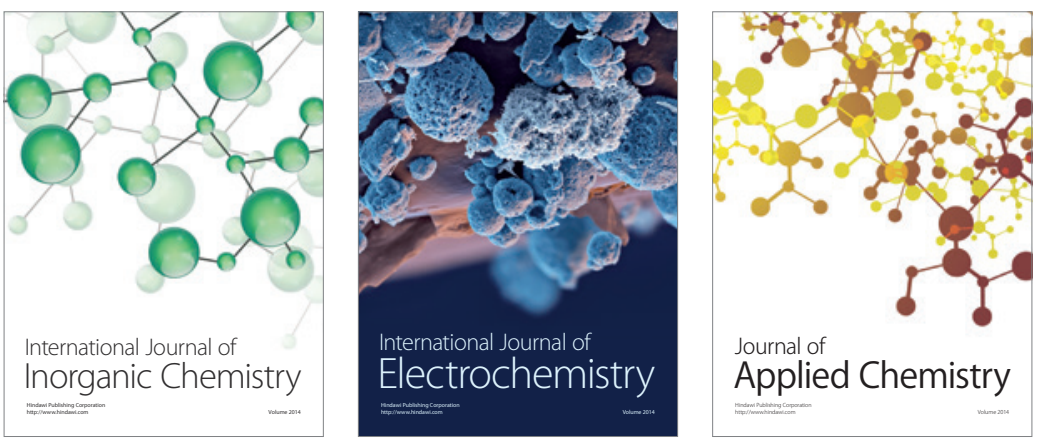

Journal of

Applied Chemistry
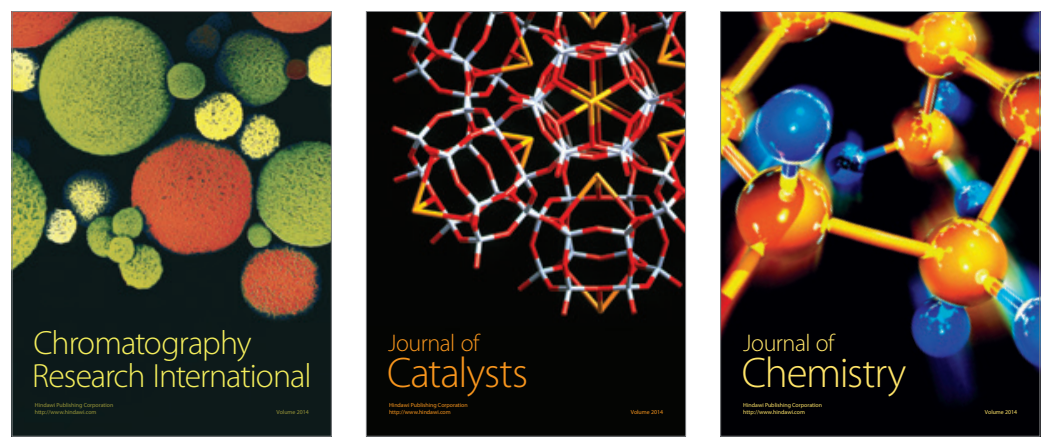
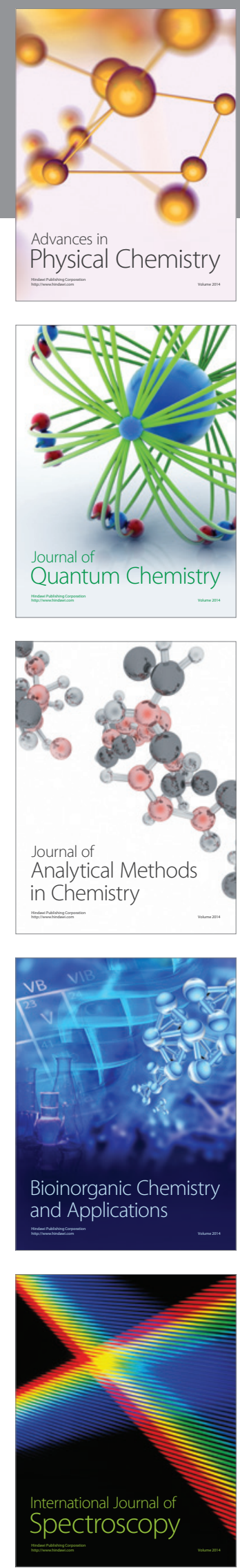